\title{
A perturbation analysis of the stability of long liquid bridges between almost circular supporting disks
}

\author{
J. Meseguer and J. M. Perales \\ IDR UPM, ETSI Aeronáwticos, Universidad Politécnica de Madrid. 28040 Madrid. Spain \\ J. I. D. Alexander \\ Department of Mechanical and Aerospace Engineering and National Center for Microgravity Research \\ on Fluids and Combustion, Case Westem Reserve University. Cleveland. Ohio 44106
}

(Received 19 April 2000; accepted 24 May 2001)

\begin{abstract}
The stability of an isothermal liquid mass of constant properties (density and surface tension) held by capillary forces between two solid disks placed a distance $L$ apart (the so-called liquid bridge model) is considered. For a weightless liquid bridge that is a right circular cylinder, the well-known Rayleigh stability limit holds. and the liquid column becomes unstable when its length is larger than its circumference. Many perturbations from this ideal configuration have been studied in the past. but the supporting disk shape has always been assumed circular. In this Brief Communication the influence of noncircular supports on stability limits of almost cylindrical liquid bridges is analyzed through an asymptotic analysis. Closed form expressions for the stability limits are presented.
\end{abstract}

Liquid bridges offer the simplest controllable liquid-gas interface that can be used to examine aspects of the fundamental plysics of capillary. Their applicability to problems involving fluid management under weightlessness has led to several experiment opportunities under the microgravity conditions that prevail both in sounding rockets and in low-earth orbit spacecraft.

The equilibrium shapes and stability limits of liquid bridges have been analyzed extensively (both theoretically and experimentally) over the last few decades. However, to our knowledge all published work to date deals with axisymmetric or non-axisymmetric liquid bridge configurations that are held between circular supports. The effects of noncircular supports have never been considered. A review ${ }^{1}$ of the state of the art in the field and a few papers ${ }^{2.3}$ concerning nonaxisymmetric liquid bridges have been published recently.

The stability of liquid bridges between almost circular supporting disks with parallel faces is analyzed here using standard bifurcation theory (Lyapunov-Schmidt technique). The fluid configuration consists of an isothermal mass of liquid of volume $\tilde{V}$ held by surface tension forces between the disks that are separated by a distance $L$. The disk shapes are given by $\tilde{F}(L / 2, \theta)$ and $\tilde{F}(-L / 2, \theta)$, respectively (see Fig. 1). The combined effect of noncircular support disks and an arbitrarily directed acceleration direction is also considered. Obviously the performed analysis reproduces some asymptotic results already known conceming the stability of either axisymmetric (coaxial circular disks with different diameters) or non-axisymmetric (e.g., non-coaxial circular disks) supporting disk configurations, but the perturbation analysis done here allows the consideration of much more general configurations.

Equilibrium shapes of liquid bridges are described by the Young-Laplace equation. In dimensional variables this takes the form $\sigma \tilde{M}(\tilde{F})+\widetilde{P}-\Delta \rho g_{a} \tilde{z}+\Delta \rho g_{i} \tilde{F} \cos \theta=0$. where $\tilde{M}(\tilde{F})$ is twice the mean curvature of the interface. $\widetilde{F}=\widetilde{F}(\widetilde{z}, \theta)$ is the equation for the liquid bridge interface, $\widetilde{P}$ is a constant related to the origin of pressure, $\Delta \rho$ is the difference in density between the liquid bridge and the surrounding fluid, $\sigma$ is the interfacial tension between the bridge and the surrounding fluid, and $g_{o}$ and $g_{l}$ are the axial and lateral components of the acceleration, respectively. The boundary conditions and constraints express the azimuthal periodicity of the interface shape. $\widetilde{F}(\widetilde{z}, \theta)=\widetilde{F}(\widetilde{z}, \theta+2 \pi)$, the constant value of the liquid bridge volume, and that the bridge surface is anchored or pinned to the edges of the

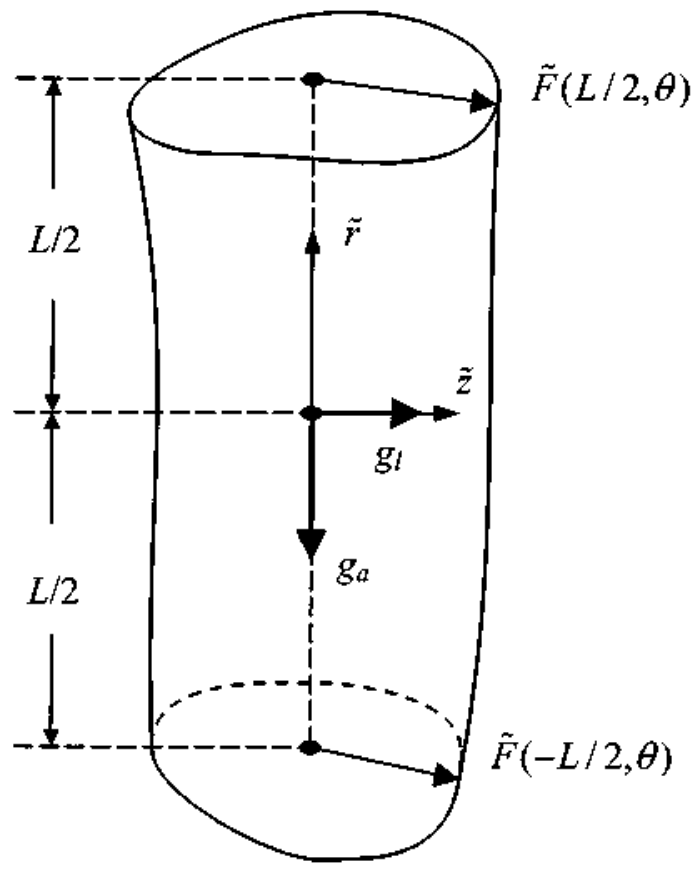

FIG. 1. The liguid bridge setup. 
supporting disks. If the following characteristic lengths are introduced:

$R^{2}=\frac{1}{2 \pi} \int_{0}^{2 \pi} \widetilde{F}(L / 2, \theta) d \theta, \quad R^{-}=\frac{1}{2 \pi} \int_{0}^{2 \pi} \widetilde{F}(-L / 2, \theta) d \theta$, then, taking $R_{0}=\left(R^{+}+R^{-}\right) / 2$ as characteristic length of the problem, and defining $F=\tilde{F} / R_{0}, z=\tilde{z} / R_{0}$, the YoungLaplace equation can be recast in dimensionless form as $M(F)+P-B_{a} z+B_{l} F \cos \theta=0$. where

$$
M(F)=\frac{F\left[1+\left(F_{z}\right)^{2}\right]\left[F_{\theta \theta}-F\right]+F F_{z z}\left[F^{2}+\left(F_{\theta}\right)^{2}\right]-2 F_{\theta}\left[F_{\theta}+F F_{z} F_{z \theta}\right]}{\left\{F^{2}\left[1+\left(F_{z}\right)^{2}\right]+\left(F_{\theta}\right)^{2}\right\}^{3 / 2}} .
$$

The dimensionless boundary conditions at the disks are

$$
F( \pm \Lambda . \theta)=\mathbf{I} \pm h+\sum_{n=1}^{\infty}\left(\gamma_{n}^{ \pm} \cos n \theta+\delta_{n}^{ \pm} \sin n \theta\right),
$$

where $h=\left(R^{+}-R^{-}\right) /\left(R^{+}+R^{-}\right)$and the sign plus must be taken for the upper disk boundary condition and the sign minus for the lower disk boundary condition in Eq. (2). The azimuthal periodicity condition is $F(z, \theta+2 \pi)=F(z, \theta)$ and the constant volume conditions take the form

$$
\frac{1}{2} \int_{-\Lambda}^{\Lambda} d z \int_{0}^{2 \pi} F^{2} d \theta=2 \pi \Lambda V
$$

Here $P$ is a constant related to the difference between the outer pressure and the inner pressure which has been made dimensionless using $\sigma / R_{0}$. In addition. $B_{a}$ is the axial Bond number, $B_{a}=\Delta \rho g_{a r} R_{0}^{2} / \sigma . B_{l}$ is the lateral bond number, $B_{l}$ $=\Delta \rho g_{l} R_{0}^{2} / \sigma, \Lambda=L /\left(2 R_{0}\right)$ is the slenderness, and $V^{\prime}$ $=\tilde{V} /\left(\pi R_{0}^{2} L\right)$ is the dimensionless volume. The subscripts $z$ and $\theta$ indicate derivatives with respect to these variables.

Critical points result after linearization of the above formulation. It is well known that for the case $B_{a}=B_{l}=h$ $=\gamma_{n}^{+}=\delta_{n}^{+}=\gamma_{n}^{-}=\delta_{n}^{-}=0 . V^{\prime}=\mathbf{I}$, the problem under consideration has the trivial equilibrium solution $F=1 . P=1$ for any $\Lambda$. The introduction of the following expansions. $F(z, \theta)$ $=1+\varepsilon \hat{f}(z, \theta)+O\left(\varepsilon^{2}\right) . P=\mathbf{I}+\varepsilon \hat{p}+O\left(\varepsilon^{2}\right)$, where $\varepsilon$ stands for the magnitude of the deformation of the interface, allows us to calculate $\hat{f}(z, \theta)$ and thus other equilibrium solutions after neglecting $O\left(\varepsilon^{2}\right)$ terms.

Nontrivial (different from zero) solutions of the linear problem occur only for a discrete number of values of $\Lambda$. The smallest value of $\Lambda$ for which a nontrivial solution (bifurcation to noncylindrical equilibrium shapes) appears is for $\Lambda$ $=\pi$. Here the transition from stable to unstable equilibrium shapes occurs (all other bifurcation points are irrelevant as they cannot be attained because the liquid bridge will break for $\Lambda>\pi$ ). Therefore, the only relevant instability appears at $\Lambda=\pi$ and. within this approximation, the departure from the cylinder of the unstable equilibrium shapes are defined by $\hat{f}(z, \theta)=\sin (\pi z / \Lambda), \hat{p}=0$, which is the solution that must be perturbed to calculate the variation of the maximum stable slenderness.

From this point on, the process used to obtain the variation of the maximum stable slendemess due to the different effects under consideration is similar to that described by
Perales $^{4}$ and Meseguer et al. ${ }^{5}$ (although the boundary conditions at the disks are now much more involved). First, a new variable, $x=\pi z / \Lambda$, which normalizes boundary conditions, is introduced along with the parameter $\lambda=1-\Lambda / \pi$, and terms of higher order than those appearing in the linear problem are retained. Let $f(x, \theta)$ and $p$ represent these higherorder terms in the expansions of the interface shape and the pressure, respectively. The new expansions for $F$ and $P$ are then $F(z, \theta)=\mathbf{I}+\varepsilon \sin x+f(x, \theta) . P=\mathbf{I}+p$. which, after substitution in the problem formulation yields the new problem

$$
\begin{gathered}
M(\mathbf{I}+\varepsilon \sin x+f(x, \theta))+\mathbf{I}+p-B_{a} x(1-\lambda) \\
+B_{i}[1+\varepsilon \sin x+f(x, \theta)] \cos \theta=0,
\end{gathered}
$$

$f( \pm \pi, \theta)= \pm h+\sum_{n=1}^{\infty}\left(\gamma_{n}^{ \pm} \cos n \theta+\delta_{n}^{ \pm} \sin n \theta\right)$,

$f(x, \theta)=f(x, \theta+2 \pi)$.

$\int_{-\pi}^{\pi} d x \int_{0}^{2 \pi}[1+\varepsilon \sin x+f(x, \theta)]^{2} d \theta=\mathbf{4} \pi^{2}(1+v)$.

where $v=V-I$ is used to characterize the difference in volume with respect to that of a cylindrical liquid bridge. The curvature of the interface, $M(F)$. is now computed in terms of the variables $x$ and $\theta$, so that $M(F)$ now includes $\lambda$ as a parameter. Note that this formulation requires an additional condition to uniquely define the parameter $\varepsilon$, that is,

$$
\int_{-\pi}^{\pi} d x \int_{0}^{2 \pi} f \sin x d \theta=0
$$

The problem defined by Eqs. (4)-(8) allows us to calculate $f(x, \theta)$ and $p$ in terms of the small parameters $\varepsilon . \lambda, h$. $B_{a}, B_{l}, \gamma_{n}^{+}, \delta_{n}^{+}, \gamma_{n}^{-}$, and $\delta_{n}^{-}$. Although a direct solution could be attempted for small values of the parameters involved, it requires that the relative orders of magnitude of the parameters be anticipated. Instead. a systematic approach based on the bifurcation equation (the Lyapunov-Schmidt technique ${ }^{6}$ ) will be used. Thus. instead of Eq. (4), the equation to be solved is

$$
\begin{aligned}
& M(\mathbf{I}+\varepsilon \sin x+f(x, \theta))+\mathbf{I}+p-B_{a} x(1-\lambda) \\
& \quad+B_{f}[1+\varepsilon \sin x+f(x, \theta)] \cos \theta+\phi \sin x=0 .
\end{aligned}
$$

From the implicit function theorem ${ }^{6}$ it can be demonstrated that. at least in a neighborhood of $\varepsilon=\lambda=v=h=B_{a}$ $=B_{f}=\gamma_{n}^{+}=\delta_{n}^{+}=\gamma_{n}^{-}=\delta_{n}^{-}=0$, the expressions (5)-(9) 
uniquely define $f, p$, and $\phi$ in terms of power series of the quoted small parameters. Such solutions will correspond to the solution of original set of equations (5)-(9) if and only if the parameters involved satisfy the bifurcation equation $\phi\left(\varepsilon, \lambda, v, h, B_{a}, B_{l}, \gamma_{n}^{+}, \delta_{n}^{+}, \gamma_{n}^{-}, \delta_{n}^{-}\right)=0$. From this point the procedure for the problem resolution is similar to that already presented by Meseguer et al., although it must be pointed out that the number of new problems to be solved becomes infinite because of the terms containing the parameters $\gamma_{n}^{+}, \delta_{n}^{+}, \gamma_{n}^{-}$, and $\delta_{n}^{-}$. However, most of these new problems have the trivial zero solution, and they do not contribute to the stability limit (additional details on the problem resolution can be obtained upon request to the authors). Once the different nonzero coefficients involved in the bifurcation equation are calculated, the bifurcation equation, $\phi=0$, reduces to

$$
\begin{aligned}
-\frac{2}{\pi} & +2 B_{a}+2 \varepsilon \lambda+\varepsilon v-\frac{1}{4 \pi} \sum_{n=1}^{\infty}\left[\left(\gamma_{n}^{+}\right)^{2}+\left(\delta_{n}^{+}\right)^{2}\right] \\
& +\frac{1}{4 \pi} \sum_{n=1}^{\infty}\left[\left(\gamma_{n}^{-}\right)^{2}+\left(\delta_{n}^{-}\right)^{2}\right]-\frac{3}{2 \pi} B_{l}\left(\gamma_{1}^{+}-\gamma_{1}^{-}\right) \\
& -\frac{\pi^{2}}{2} \varepsilon B_{l}^{2}-\frac{3}{2} \varepsilon^{3}+\cdots=0,
\end{aligned}
$$

where only the leading terms for each parameter have been written. The stability limit is defined by $\lambda=\lambda_{\operatorname{mm}}$, or $\Lambda_{\max }$ $=\pi\left(1-\lambda_{\operatorname{mm}}\right)$. Thus, taking $d \lambda / d \varepsilon=0$, we find that to leading order $\Lambda_{\max }$ is given by

$$
\begin{aligned}
\Lambda_{\max }= & \pi\left\{1-\left(\frac{3}{2}\right)^{4 / 3}\right. \\
& \times\left[B_{a}-\frac{h}{\pi}-\frac{1}{8 \pi}\left(\Gamma^{+}-\Gamma^{-}\right)-\frac{3}{4 \pi} B_{l}\left(\gamma_{1}^{+}-\gamma_{1}^{-}\right)\right]^{2 / 3} \\
& \left.+\frac{1}{2} v-\frac{\pi^{2}}{4} B_{l}^{2}\right\}
\end{aligned}
$$

where

$$
\begin{aligned}
\Gamma^{+} & =\sum_{n=1}^{\infty}\left[\left(\gamma_{n}^{+}\right)^{2}+\left(\delta_{n}^{+}\right)^{2}\right] \\
& =\frac{1}{\pi} \int_{0}^{2 \pi}(F( \pm \Lambda, \theta)-1-h)^{2} d \theta, \\
\Gamma^{-} & =\sum_{n=1}^{\infty}\left[\left(\gamma_{n}^{-}\right)^{2}+\left(\delta_{n}^{-}\right)^{2}\right] \\
& =\frac{1}{\pi} \int_{0}^{2 \pi}(F( \pm \Lambda, \theta)-1+h)^{2} d \theta .
\end{aligned}
$$

The quantitative influence of the different parameters is determined by the exponents of each group of terms in $\mathrm{Eq}$. (10) and can be understood by considering the effect of the imposed perturbation on the necking of the liquid column. The expression for both stable and unstable equilibrium interface shapes is of the form $f(x)=1+\varepsilon \sin x$. When the instability develops, the liquid bridge interface bulges in one half of the liquid column and necks in the other (the instability is antisymmetric with respect to the mid-plane parallel to the disks). According to this behavior, any perturbation leading to an antisymmetric deformation of the interface will decrease the stability limit. Note that the reduction of the maximum stable slenderness is proportional to the two-thirds power of the perturbation. Perturbations that cause a symmetric deformation (with respect to the mid-plane) of the interface, such as those due to a reduction in volume or to the presence of lateral Bond number, may also reduce the stability limit. However these kinds of perturbation are less critical and now the reduction in $\Lambda_{\max }$ is either linear in the volume. $v$, or depends on the square of the lateral Bond number.

To further illustrate the liquid bridge behavior predicted by $\mathrm{Eq}$. ( 10 ), let us assume a liquid bridge of cylindrical volume ( $v=0$ ) subject only to a small axial Bond number $\left(B_{a}\right.$ $\neq 0, B_{l}=0$ ) such that the acceleration is constant and is coincident with the liquid bridge axis. If the axial Bond number is positive, the liquid column will bulge at the lower half and it will neck at the upper half. In the vicinity of the stability limit, the interface deformation is calculated by first solving for $\bar{\varepsilon}$ the equation:

$$
-\frac{1}{\pi} h+B_{a}+\overline{\varepsilon \lambda}-\frac{3}{4} \overrightarrow{\varepsilon^{3}}=0 .
$$

This equation has three roots for $\lambda>\lambda_{\operatorname{mm}}$ as shown in Fig. 2. The root represented by the solid line in Fig. 2 is the root with the smallest $|\vec{\varepsilon}|$ for $\lambda>0$ and corresponds to the stable solution. Note that, $\bar{\varepsilon}<0$ for $B_{a}>0, h=0$ and $\bar{\varepsilon}>0$ for $B_{a}=0 . h>0$.

The interface shape is given by $F(x, \theta)=1+\bar{\varepsilon} \sin x$ (the term in $\bar{\varepsilon}$ is of the order of $B_{a}^{1 ; 3}$ or $h^{1 / 3}$ and thus the dominant term in the expansion). To the order considered here, the magnitude of the interface deformation could also be measured by the excess of liquid volume with respect to the cylindrical one in the lower part of the bridge (or the volume deficiency in the upper part). The excess volume is given by

$$
\Delta v=\bar{\varepsilon} \int_{-\pi}^{0} d x \int_{0}^{2 \pi} \sin x d \theta=-2 \pi \bar{\varepsilon} .
$$

Note that $h>0$ (larger disk at the top) decreases the volume at the lower half of the column. whereas a positive Bond number increases it. This explains why this configuration is more stable than the opposite configuration where the larger disk is at the bottom. The reason for this is that when the large disk is at the bottom. a positive Bond number tends to increase the volume in the lower half of the liquid bridge. Now for unequal radii disks. the configuration with the smaller disk at the bottom will have a lower volume. The effect of having a large disk at the bottom together with a positive Bond number tends to enhance the tendency of the liquid column to neck in the upper half of the column, ${ }^{7,8}$ and the stability limit decreases.

If solid supports have noncircular shape, the same reasoning applies. The liquid bridge interface must fulfill the pinning condition at the disk edges. Thus, for a given disk area the perimeter is a minimum when the disks are circular. 

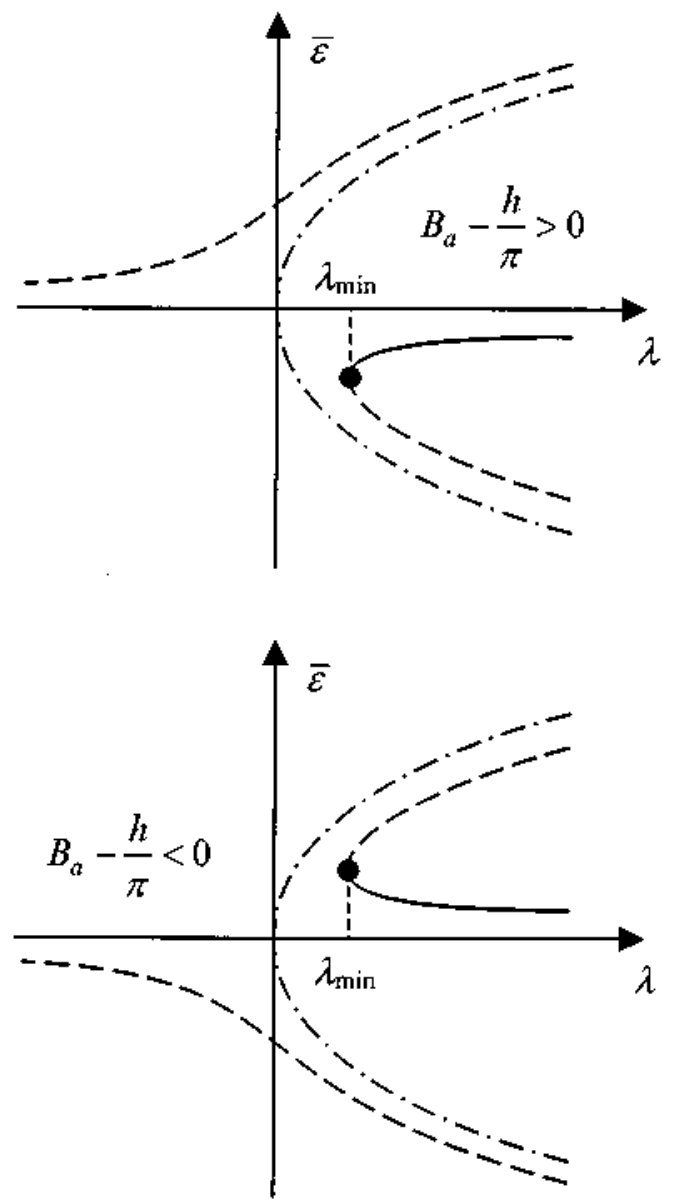

FIG. 2. Sketch of the equilibrium solution (departure from the cylinder $\bar{\varepsilon}$ ) as a function of $\lambda$. The stable solution is plotted as a solid line and the unstable solutions as a dashed line.

Consequently, any disk shape different from the circular one means that there will be an excess of liquid volume near this disk and hence, the appearance of a necking effect at the far disk. For example, assume that the upper disk has an elliptical shape, but only slightly deviates from an almost circle, with major and minor semi-axes equal to $\mathbf{I}+a$ and $\mathbf{I}-a$, respectively. with $a \ll 1$. That is. $\tilde{F}(L / 2, \theta)=\left(1-a^{2}\right)\left(1+a^{2}\right.$ $-2 a \cos 2 \theta)^{-1 / 2} \approx 1+a \cos 2 \theta+O\left(a^{2}\right)$, then $R^{+}=1$ and $F(\Lambda, \theta)=1+\alpha \cos 2 \theta+O\left(\alpha^{2}\right)$, with $\alpha=a / R^{+}$, so that $\Gamma^{+}$ $=\alpha^{2}+O\left(\alpha^{4}\right)$. Within this approximation an upper elliptical disk enlarges the stability margin of a liquid bridge subjected to an axial positive Bond number. In particular. for this case. Eq. (11) now reads

$$
\Lambda_{\max }=\pi\left\{1-\left(\frac{3}{2}\right)^{4 / 3}\left[B_{a}-\frac{1}{8 \pi} \alpha^{2}\right]^{2 / 3}\right\} .
$$

Note that it would even be possible to stabilize an otherwise unstable liquid bridge by offsetting the destabilizing effect of the axial Bond number by choosing the appropriate ellipse eccentricity $\left[\alpha=\left(8 \pi B_{a}\right)^{1 / 2}\right]$.

Another interesting configuration occurs when both disks are elliptical. In this case the expression for the lower disk is $\tilde{F}(-L / 2, \theta)=\left(1-a^{2}\right)\left(1+a^{2}-2 a \cos 2(\theta+\varphi)\right)^{-1 / 2}$ $\approx 1+a \cos 2(\theta+\varphi)+O\left(a^{2}\right)$, where $\varphi$ is the angle between the major axes of the upper and lower disks. Thus, $F(-\Lambda, \theta)$ $=1+\alpha \cos 2 \varphi \cos 2 \theta-\alpha \sin 2 \varphi \sin 2 \theta+O\left(\alpha^{2}\right)$, and Eq. (13) yields $\Gamma^{-}=\alpha^{2}+O\left(\alpha^{4}\right)$, regardless of the value of $\varphi$. It then follows that, since in this case $\Gamma^{+}=\Gamma^{-}$, to this order the stability limit is independent of whether the disks are elliptic, irrespective of their relative angular position. This conclusion holds for any other disk shape.

In conclusion, the stability of liquid bridges with an almost cylindrical volume, spanning between noncircular supporting disks. has been analyzed by using standard bifurcation techniques. The analysis shows that to the order considered here noncircular disks effects modify liquid bridge stability in a way qualitatively similar to other nonsymmetric effects (with respect to the mid-plane) like axial gravity or disks having unequal diameter.

The results obtained show that when a noncircular disk is the only effect considered the maximum stable length of the column is reduced (as one could expect), but when it is combined with other nonsymmetric effects, the maximum stable length of the bridge depends on the combination of the different effects considered, and there can be situations in which the resulting configuration is more stable than it would be with only one perturbation. This happens when two nonsymmetric effects act simultaneously with opposite senses. When both disks are equal in shape (either circular or noncircular). to the leading order considered here. the stability limit does not change no matter the relative orientation of one of the disks with respect to the other. Higher approximations should be considered to elucidate how this twisting effect affects (marginally) the liquid bridge stability.

\section{ACKNOWLEDGMENTS}

This work has been supported by the Spanish Ministerio de Ciencia y Tecnología (PNE-002/2001-C), and by NASA through Grants No. NAG3-1864 and No. NAG3-2160.

\footnotetext{
${ }^{1}$ J. Meseguer, J. M. Perales. I. Martinez, N. A. Bezdenejnykh, and A. Sanz, "Hydrostatic instabilities in floating zone crystal growth process," Curr. Top. Cryst. Growth Res. 5. 27 (1999).

${ }^{2}$ N. A. Bezdenejuykh, J. Meseguer, and J. M. Perales, "An experimental analysis of the instability of non-axisymmetric litjuid bridges in a gravitational field," Phys. Fluids 11, 3181 (1999).

${ }^{3}$ F. Zayas, J. I. D. Alexander, J. Meseguer, and J.-F. Ramus, "On the stability linits of long non-axisymmetric cylindrical liquid bridges," Phys. Fluids 12, 979 (2000).

${ }^{4} \mathrm{~J}$. M. Perales, "Non-axisymmetric effects on long liquid bridges," Acta Astron. 15, 561 (1987).

${ }^{5} \mathrm{~J}$. Meseguer, N. A. Bezdenejuykh, J. M. Perales, and P. Rodriguez de Franciseo, "Theoretical and experimental analysis of stability limits of non-axisymmetric liquid bridges under microgravity conditions," Microgravity Sci. Teclunol, 8. 2 (1995).

${ }^{6}$ N. S. Chow and J. K. Hale, Methods of Bifircation Theory (SpringerVerlag. Berlin, 1982).

${ }^{7} \mathrm{~J}$. Meseguer, "Stability of slender, axisymmetric liquid bridges between unequal disks," J. Cryst. Growth 67, 141 (1984).

${ }^{8}$ L. A. Slobozhanio and J. L. D. Alexander, "Combined effect of dise inequality and axial gravity on axisymmetric liquid bridge stability," Phys. Fluids 10, 2473 (1998).
} 